\title{
A CONFORMAL DIFFERENTIAL INVARIANT AND THE CONFORMAL RIGIDITY OF HYPERSURFACES
}

\author{
MAKS A. AKIVIS AND VLADISLAV V. GOLDBERG \\ (Communicated by Christopher Croke)
}

\begin{abstract}
For a hypersurface $V^{n-1}$ of a conformal space, we introduce a conformal differential invariant $I=\frac{h^{2}}{g}$, where $g$ and $h$ are the first and the second fundamental forms of $V^{n-1}$ connected by the apolarity condition. This invariant is called the conformal quadratic element of $V^{n-1}$. The solution of the problem of conformal rigidity is presented in the framework of conformal differential geometry and connected with the conformal quadratic element of $V^{n-1}$. The main theorem states:

Let $n \geq 4$, and let $V^{n-1}$ and $\bar{V}^{n-1}$ be two nonisotropic hypersurfaces without umbilical points in a conformal space $C^{n}$ or a pseudoconformal space $C_{q}^{n}$ of signature $(p, q), \quad p=n-q$. Suppose that there is a one-to-one correspondence $f: V^{n-1} \rightarrow \bar{V}^{n-1}$ between points of these hypersurfaces, and in the corresponding points of $V^{n-1}$ and $\bar{V}^{n-1}$ the following condition holds: $\bar{I}=f_{*} I$, where $f_{*}: T\left(V^{n-1}\right) \rightarrow T\left(\bar{V}^{n-1}\right)$ is a mapping induced by the correspondence $f$. Then the hypersurfaces $V^{n-1}$ and $\bar{V}^{n-1}$ are conformally equivalent.
\end{abstract}

1. In local differential geometry the rigidity theorems contain conditions under which two submanifolds of a homogeneous space can differ only by a location in the space. For hypersurfaces in a projective space, the rigidity problem was considered by G. Fubini [6, 7] (see also pp. 605-629 of the book [8] by Fubini and E. Cech), É. Cartan [5], G. Jensen and E. Musso [10], and by the authors of this paper in the book [1] (Section 7.4).

The problem of conformal rigidity of submanifolds is also of great interest. This problem was studied by Cartan [4], M. do Carmo and M. Dajczer [2] and R. Sacksteder [11] (see also the paper [13] by R. Sulanke in which the author considered problems close to the rigidity problem). However, in these papers the problem of conformal rigidity was investigated in the framework of Euclidean geometry.

In the current paper we present the solution of this problem in the framework of conformal differential geometry. To this end, we introduce a conformal quadratic element and prove that if $n \geq 4$ and there exists a one-to-one point correspondence of two hypersurfaces both not having umbilical points preserving this quadratic

Received by the editors November 21, 1995 and, in revised form, February 23, 1996.

1991 Mathematics Subject Classification. Primary 53A30.

Key words and phrases. Conformal and pseudoconformal geometry, hypersurface, first and second fundamental forms, conformal quadratic element, moving frames, conformal rigidity.

This research was partially supported by Volkswagen-Stiftung (RiP-program at MFO). The research of the first author was also partially supported by the Israel Ministry of Absorption and the Israel Public Council for Soviet Jewry. 
element, then the hypersurfaces are conformally equivalent. Moreover, we consider the rigidity problem not only for hypersurfaces of a conformal space but also for hypersurfaces of a pseudoconformal space. We only assume that a hypersurface is not isotropic (i.e., its tangent subspaces are not tangent to the isotropic cones).

2. Let $V^{n-1}$ be a nonisotropic hypersurface of a real conformal space $C^{n}$ or a real pseudoconformal space $C_{q}^{n}$ of signature $(p, q)$, where $p=n-q$. With any point $x \in V^{n-1}$, we associate a conformal moving frame consisting of two points $A_{0}=x$ and $A_{n+1}$ and $n$ independent hyperspheres $A_{1}, \ldots, A_{n}$, passing through these two points. We will assume that the hypersphere $A_{n}$ is tangent to the hypersurface $V^{n-1}$ at the point $A_{0}$, and the hyperspheres $A_{i}, i=1, \ldots, n-1$, are orthogonal to $V^{n-1}$ at the point $A_{0}$. Then the frame elements satisfy the following conditions:

$$
\begin{array}{ll}
\left(A_{0}, A_{0}\right)=\left(A_{n+1}, A_{n+1}\right)=0, & \left(A_{0}, A_{i}\right)=\left(A_{n+1}, A_{i}\right)=0, \\
\left(A_{0}, A_{n}\right)=\left(A_{n+1}, A_{n}\right)=0, & \left(A_{i}, A_{n}\right)=0,
\end{array}
$$

where the parentheses denote the scalar product of corresponding frame elements. The first two of these conditions mean that the frame elements $A_{0}$ and $A_{n+1}$ are points, the following four conditions mean that the hyperspheres $A_{i}$ and $A_{n}$ pass through these two points, and finally, the last condition expresses the orthogonality of the hyperspheres $A_{i}$ and $A_{n}$. In addition, we normalize the points $A_{0}$ and $A_{n+1}$ by the condition

$$
\left(A_{0}, A_{n+1}\right)=-1 .
$$

We will not demand the orthogonality of the hyperspheres $A_{i}$ and will write their scalar products in the form:

$$
\left(A_{i}, A_{j}\right)=g_{i j},
$$

where $\operatorname{det}\left(g_{i j}\right) \neq 0$, since the hypersurface $V^{n-1}$ is not isotropic.

If $X$ is an arbitrary point of the space $C^{n}$ or the space $C_{q}^{n}$, then it can be represented as

$$
X=x^{0} A_{0}+x^{i} A_{i}+x^{n} A_{n}+x^{n+1} A_{n+1} .
$$

Since for any point $X \in C^{n}$, we have $(X, X)=0$, then it follows that the coordinates of the point $X$ satisfy the equation

$$
g_{i j} x^{i} x^{j}+\epsilon\left(x^{n}\right)^{2}-2 x^{0} x^{n+1}=0,
$$

where $\epsilon=\left(A_{n}, A_{n}\right) \neq 0$, since the quadratic form on the left-hand side of the last equation is nondegenerate. The last equation is the equation of a nondegenerate hyperquadric $Q^{n}$ of a projective space $P^{n+1}$ onto which the space $C^{n}$ or the space $C_{q}^{n}$ is mapped under the Darboux mapping. The left-hand side of this equation is of signature $(p+1, q+1)$. Under the Darboux mapping, the images of the points $A_{0}$ and $A_{n+1}$ are the points lying on the hyperquadric $Q^{n}$, and in general, the images of the hyperspheres $A_{i}$ and $A_{n}$ are the points not belonging to $Q^{n}$.

We will prove now that the quantity $\epsilon$ can be always reduced to 1 . In fact, if $\epsilon>0$, then this can be achieved by means of renormalization of the hypersphere $A_{n}$. If $\epsilon<0$, then we can replace the point $A_{0}$ by $-A_{0}$ which implies

$$
g_{i j} x^{i} x^{j}+\epsilon\left(x^{n}\right)^{2}+2 x^{0} x^{n+1}=0
$$

and then change the sign of the left-hand side of the above equation and again reduce to 1 the positive quantity $-\epsilon$. As a result, the equation of the Darboux 
hyperquadric takes the form

$$
-g_{i j} x^{i} x^{j}+\left(x^{n}\right)^{2}-2 x^{0} x^{n+1}=0 .
$$

By setting $g_{i j}=-\widetilde{g}_{i j}$ and suppressing tilde, we reduce the last equation to the form

$$
g_{i j} x^{i} x^{j}+\left(x^{n}\right)^{2}-2 x^{0} x^{n+1}=0 .
$$

Therefore, for any $\epsilon \neq 0$, we can normalize the hypersphere $A_{n}$ in such a way that

$$
\left(A_{n}, A_{n}\right)=1 .
$$

The form $g_{i j} x^{i} x^{j}$ in equation (4) has signature $(p-1, q)$.

3. The equations of infinitesimal displacement of our conformal frame have the form:

$$
d A_{\xi}=\omega_{\xi}^{\eta} A_{\eta}, \quad \xi, \eta=0,1, \ldots, n+1,
$$

where $\omega_{\xi}^{\eta}$ are 1-forms containing parameters, on which the family of frames in question depends, and their differentials: $\omega_{\xi}^{\eta}=\omega_{\xi}^{\eta}(u, d u)$. By equations (1)-(3) and (5), these forms must satisfy the following conditions:

$$
\begin{cases}\omega_{0}^{n+1}=\omega_{n+1}^{0}=0, & \omega_{0}^{0}+\omega_{n+1}^{n+1}=0, \\ \omega_{i}^{n+1}=g_{i j} \omega_{0}^{j}, & \omega_{n+1}^{i}=g^{i j} \omega_{j}^{0}, \\ \omega_{n}^{n+1}=\omega_{0}^{n}, & \omega_{n+1}^{n}=\omega_{n}^{0}, \\ \omega_{i}^{n}=-g_{i j} \omega_{n}^{j}, & \omega_{n}^{n}=0, \\ d g_{i j}=g_{i k} \omega_{j}^{k}+g_{k j} \omega_{i}^{k} . & \end{cases}
$$

In addition, the forms $\omega_{\xi}^{\eta}$ satisfy the structure equations of the conformal space:

$$
d \omega_{\xi}^{\eta}=\omega_{\xi}^{\zeta} \wedge \omega_{\zeta}^{\eta}, \quad \xi, \eta, \zeta=0,1, \ldots, n+1 .
$$

4. Since the hypersphere $A_{n}$ is tangent to the hypersurface $V^{n-1}$ at the point $x=A_{0}$, the condition $\left(A_{n}, d A_{0}\right)=0$ holds. It follows from this condition that

$$
\omega_{0}^{n}=0 .
$$

This equation determines the family of frames of first order associated with the hypersurface $V^{n-1}$. This family can be considered as the frame bundle $\mathcal{R}^{1}\left(V^{n-1}\right)$ of first order with the base $V^{n-1}$. Its fiber is a set of frames which is associated with the point $x \in V^{n-1}$ in the manner indicated above. The structure group of the frame bundle $\mathcal{R}^{1}\left(V^{n-1}\right)$ is a subgroup of the fundamental group of the space $C_{q}^{n}$ whose transformations leave invariant the tangent element of $V^{n-1}$ consisting of a point $x \in V^{n-1}$ and the tangent subspace $T_{x}\left(V^{n-1}\right)$. The 1 -forms $\omega_{0}^{i}$, which we will denote further by $\omega^{i}$, are basis forms of the frame bundle $\mathcal{R}^{1}\left(V^{n-1}\right)$, and the 1-forms $\omega_{0}^{0}, \omega_{i}^{0}, \omega_{n}^{0}$, and $\omega_{j}^{i}$ are its fiber forms.

By equation (9), on the hypersurface $V^{n-1}$, we have

$$
d A_{0}=\omega_{0}^{0} A_{0}+\omega^{i} A_{i} .
$$

By virtue of this,

$$
\left(d A_{0}, d A_{0}\right)=g_{i j} \omega^{i} \omega^{j} .
$$

The quadratic form $g=g_{i j} \omega^{i} \omega^{j}$ is relatively invariant and determines a conformal structure on the hypersurface $V^{n-1}$. This form is nondegenerate and of signature $(p-1, q)$. For $q=0$ (i.e., for the proper conformal space $C^{n}$ ) the form $g$ is positive 
definite. For $p=1$ and $q=n-1$ (i.e., for a conformal space $C_{1}^{n}$ of Lorentzian signature) there exist hypersurfaces with signature $(0, q)$. Such hypersurfaces are called spacelike, and a conformal structure induced on them is properly conformal. The equation $g_{i j} \omega^{i} \omega^{j}=0$ determines the isotropic cone of the hypersurface $V^{n-1}$.

Taking the exterior derivative of equation (9) and applying (8), we obtain

$$
\omega^{i} \wedge \omega_{i}^{n}=0
$$

Applying Cartan's lemma to equation (11), we find that

$$
\omega_{i}^{n}=\lambda_{i j} \omega^{j}, \quad \lambda_{i j}=\lambda_{j i} .
$$

Differentiating equation (10), we obtain

$$
\begin{aligned}
d^{2} A_{0}= & \left(d \omega_{0}^{0}+\left(\omega_{0}^{0}\right)^{2}+\omega_{0}^{i} \omega_{i}^{0}\right) A_{0}+\left(\omega_{0}^{0} \omega_{0}^{i}+\omega_{0}^{j} \omega_{j}^{i}\right) A_{i} \\
& +\omega_{0}^{i} \omega_{i}^{n} A_{n}+\omega_{0}^{i} \omega_{i}^{n+1} A_{n+1} .
\end{aligned}
$$

It follows that

$$
\bar{h}=\left(d^{2} A_{0}, A_{n}\right)=\omega^{i} \omega_{n}^{i}=\lambda_{i j} \omega^{i} \omega^{j} .
$$

In the tangent subspace $T_{x}\left(V^{n-1}\right)$, the equation $\bar{h}=0$ determines the cone of directions along which the hypersphere $A_{n}$ has a second order tangency with $V^{n-1}$.

But the quadratic form $\bar{h}$ is not conformally invariant since all hyperspheres of the pencil

$$
A_{n}^{\prime}=A_{n}+s A_{0}
$$

as well as the hypersphere $A_{n}$ are tangent to the hypersurface $V^{n-1}$. In view of this, we obtain the following pencil of quadratic forms:

$$
\left(d^{2} A_{0}, A_{n}^{\prime}\right)=\omega_{0}^{i} \omega_{i}^{n}-s \omega_{0}^{i} \omega_{i}^{n+1}=\left(\lambda_{i j}-s g_{i j}\right) \omega^{i} \omega^{j},
$$

and all quadratic forms of this pencil have equal rights. From this pencil we will distinguish one form:

$$
h=h_{i j} \omega^{i} \omega^{j}
$$

where

$$
h_{i j}=\lambda_{i j}-\lambda g_{i j} \text { and } \lambda=\frac{1}{n-1} \lambda_{i j} g^{i j}
$$

It is easy to see that the coefficients $h_{i j}$ satisfy the apolarity condition (the trace-free condition):

$$
h_{i j} g^{i j}=0,
$$

where $g^{i j}$ is the inverse tensor for $g_{i j}$.

One can prove that geometrically condition (13) means that the cone determined by the equation $h=0$ is real and there exists an orthogonal $(n-1)$-hedron formed by tangent directions to $V^{n-1}$ at the point $x$ which is inscribed into this cone (see [1], pp. 214-216).

The tangent hypersphere $C_{n}=A_{n}+\lambda A_{0}$ is conformally invariant. It is called the central tangent hypersphere. The cone $h=0$ is composed of directions along which the hypersphere $C_{n}$ has a second order tangency with $V^{n-1}$.

The quadratic forms $g$ and $h$ are called the first and second fundamental forms of the hypersurface $V^{n-1}$.

Points of the hypersurface $V^{n-1}$, in which the forms $\bar{h}$ and $g$ are proportional, are called umbilical points. It is well-known that if at any point $x \in V^{n-1}$, we have 
$\bar{h}=\kappa g$, then the hypersurface $V^{n-1}$ is a hypersphere or its open part. Note that by (13) the condition $\bar{h}=\kappa g$ implies $h=0$.

If in the frame $\mathcal{R}_{x}\left(V^{n-1}\right)$ associated with a point $x \in V^{n-1}$ we replace the tangent hypersphere $A_{n}$ by the central tangent hypersphere $C_{n}$, then we obtain a second order frame $\mathcal{R}_{x}^{2}\left(V^{n-1}\right) \subset \mathcal{R}^{2}\left(V^{n-1}\right)$. Transformations of the structural group of the fibre bundle $\mathcal{R}^{2}\left(V^{n-1}\right)$ leave invariant not only the tangent element of the hypersurface $V^{n-1}$ but also the central tangent hypersphere $C_{n}$ attached to a point $x \in V^{n-1}$. In the fiber bundle $\mathcal{R}^{2}\left(V^{n-1}\right)$, the number of fiber forms will be reduced, since the 1-form $\omega_{n}^{0}$ becomes a linear combination of the basis forms $\omega^{i}$.

With respect to a second order frame, equation (12) takes the form

$$
\omega_{i}^{n}=h_{i j} \omega^{j} .
$$

The point $A_{0} \in V^{n-1}$ admits the normalization

$$
A_{0}^{\prime}=r A_{0}, \quad r \neq 0 \text {. }
$$

In order to preserve condition (2), we also normalize the point $A_{n+1}$ as follows: $A_{n+1}^{\prime}=\frac{1}{r} A_{n+1}$. Since under this normalization we have

$$
\left(d A_{0}^{\prime}, d A_{0}^{\prime}\right)=r^{2}\left(d A_{0}, d A_{0}\right)
$$

the quadratic form $g$ undergoes the transformation

$$
g^{\prime}=r^{2} g
$$

Moreover, we have

$$
\left(d^{2} A_{0}^{\prime}, C_{n}\right)=r\left(d^{2} A_{0}, C_{n}\right)
$$

and as a result,

$$
h^{\prime}=r h .
$$

It follows from relations (16) and (17) that the fundamental forms $g$ and $h$ of the hypersurface $V^{n-1}$ are relatively invariant forms of weights 2 and 1 , respectively.

The forms $g$ and $h$ allow us to construct the expression

$$
I=\frac{h^{2}}{g}
$$

which is invariant with respect to normalization (15). Hence, this expression is an absolute conformal invariant of the hypersurface $V^{n-1}$. It is determined in a second differential neighborhood of $V^{n-1}$. The expression $I=I\left(x, \omega^{i}\right)$ is a homogeneous function of second order with respect to the basic forms $\omega^{i}$ of the hypersurface $V^{n-1}$. We will call this function the conformal quadratic element of the hypersurface $V^{n-1}$ (cf. with the projective linear element of $V^{n-1} \subset P^{n}$ considered in [6], [7], [8], [10], [1]). At umbilical points, the invariant $I$ vanishes, since $h=0$ at these points.

Since the invariant $I$ is defined by means of the first and second quadratic fundamental forms the hypersurface $V^{n-1}$, it is a second order invariant.

Lemma. For $n \geq 4$, at unumbilical points of the hypersurface $V^{n-1}$, the conformal quadratic element $I$ is not a quadratic form with respect to $\omega^{i}$.

Proof. Suppose that

$$
\frac{h^{2}}{g}=\theta,
$$


where $\theta=\theta_{i j} \omega^{i} \omega^{j}$ is a quadratic form, that is,

$$
h^{2}=g \theta .
$$

Suppose that rank $h=\rho$. We have $\rho \neq 0$, since the points under consideration are not umbilical. Moreover, we have $\rho \neq 1$, since if $\rho=1$, then there exists a coordinate system in which all components of the tensor $h_{i j}$ vanish except $h_{11}$. But then condition (13) reduces to $h_{11} g^{11}=0$. Since $g^{11} \neq 0$, it follows that $h_{11}=0$ and $\rho=0$. Consider the cases $\rho=2$ and $\rho>2$ separately. For $\rho=2$, the form $h$ can be always written as

$$
h=\alpha \cdot \beta,
$$

where $\alpha$ and $\beta$ are linear forms with respect to $\omega^{i}$, since by (13) the form $h$ is alternating. Thus, from equation (19) it follows that the quadratic form $g$ is also decomposable. However, this is impossible since the form $g$ is nondegenerate and for $n \geq 4$, is not decomposable into the product of linear factors.

If $\rho>2$, then the forms $g$ and $h$ are not decomposable, and hence equation (19) is possible only if $g$ and $h$ are proportional, that is, only at umbilical points.

5. Consider two smooth, oriented, connected and simply connected hypersurfaces $V^{n-1}$ and $\bar{V}^{n-1}$ of a conformal space $C^{n}$ or a pseudoconformal space $C_{q}^{n}$. Suppose that there is a one-to-one correspondence $f: V^{n-1} \rightarrow \bar{V}^{n-1}$ under which $f(x)=\bar{x}$, where $x \in V^{n-1}$ and $\bar{x} \in \bar{V}^{n-1}$. The correspondence $f$ induces a mapping $f_{*}$ of the tangent bundle $T\left(V^{n-1}\right)$ onto the tangent bundle $T\left(\bar{V}^{n-1}\right)$ : $f_{*}: T\left(V^{n-1}\right) \rightarrow T\left(\bar{V}^{n-1}\right)$ such that $\left.f_{*}\right|_{V^{n-1}}=f$ and $f_{*} \mid T_{x}\left(V^{n-1}\right)$ is a linear nondegenerate mapping.

We will prove now the theorem on conformal rigidity of hypersurfaces.

Theorem. Let $n \geq 4$, and $V^{n-1}$ and $\bar{V}^{n-1}$ be two nonisotropic hypersurfaces without umbilical points in a real conformal space $C^{n}$ or a real pseudoconformal space $C_{q}^{n}$. Suppose that there is a one-to-one correspondence $f: V^{n-1} \rightarrow \bar{V}^{n-1}$ between points of these hypersurfaces, and in the corresponding points of $V^{n-1}$ and $\bar{V}^{n-1}$ the following condition holds:

$$
\bar{I}=f_{*} I,
$$

where $I$ and $\bar{I}$ are the conformal quadratic elements of the hypersurfaces $V^{n-1}$ and $\bar{V}^{n-1}$ defined above. Then the hypersurfaces $V^{n-1}$ and $\bar{V}^{n-1}$ are conformally equivalent, i.e., there exists a conformal transformation $\varphi$ of the space $C^{n}$ or $C_{q}^{n}$ such that $\varphi\left(V^{n-1}\right)=\bar{V}^{n-1}$.

Proof. Relation (20) can be written in the form

$$
\frac{\bar{h}^{2}}{\bar{g}}=\frac{h^{2}}{g},
$$

from which it follows that

$$
g \cdot \bar{h}^{2}=\bar{g} \cdot h^{2} .
$$

Here and in what follows, for simplicity, we write $g$ and $h$ instead of $f_{*} g$ and $f_{*} h$. By the above lemma, each pair of the forms $\bar{h}^{2}, \bar{g}$ and $h^{2}, g$ do not have common 
factors. It follows that

$$
\bar{g}=\sigma^{2} g, \bar{h}=\sigma h,
$$

where $\sigma=\sigma(x) \neq 0$.

Further, let $x \in V^{n-1}$ and $\bar{x} \in \bar{V}^{n-1}$ be two corresponding points of the hypersurfaces $V^{n-1}$ and $\bar{V}^{n-1}$, and let $\varphi$ be a conformal transformation mapping $x=A_{0}$ into $\bar{x}=\bar{A}_{0}$, the central tangent hypersphere $C_{n}$ into the central tangent hypersphere $\bar{C}_{n}$, and the normal hyperspheres $A_{i}$ into the normal hyperspheres $\bar{A}_{i}$. Then the equations of $V^{n-1}$ and $\bar{V}^{n-1}$ have the form

$$
\omega_{0}^{n}=0, \bar{\omega}_{0}^{n}=0 .
$$

Moreover, the basis forms of $V^{n-1}$ and $\bar{V}^{n-1}$ are equal:

$$
\bar{\omega}_{0}^{i}=\omega_{0}^{i} .
$$

Since the first fundamental forms of the hypersurfaces $V^{n-1}$ and $\bar{V}^{n-1}$ have the form

$$
g=g_{i j} \omega^{i} \omega^{j}, \quad \bar{g}=\bar{g}_{i j} \omega^{i} \omega^{j}
$$

and their second fundamental forms have the form

$$
h=h_{i j} \omega^{i} \omega^{j}, \quad \bar{h}=\bar{h}_{i j} \omega^{i} \omega^{j},
$$

where $g^{i j} h_{i j}=0$ and $\bar{g}^{i j} \bar{h}_{i j}=0$ (see (13)), it follows that relations (22) are equivalent to the relations

and

$$
\bar{g}_{i j}=\sigma^{2} g_{i j}
$$

Since $\sigma \neq 0$, then by renormalizing the point $A_{0}$, this factor can be reduced to 1. In fact, setting

we find that

$$
\bar{A}_{0}^{\prime}=\frac{1}{\sigma} \bar{A}_{0},
$$

$$
d \bar{A}_{0}^{\prime}=d\left(\frac{1}{\sigma}\right) \bar{A}_{0}+\frac{1}{\sigma}\left(\bar{\omega}_{0}^{0} \bar{A}_{0}+\omega^{i} \bar{A}_{i}\right)
$$

and

Thus, we obtained

$$
\left(d \bar{A}_{0}^{\prime}, d \bar{A}_{0}^{\prime}\right)=\frac{1}{\sigma^{2}} \bar{g}_{i j} \omega^{i} \omega^{j}=g_{i j} \omega^{i} \omega^{j} .
$$

$$
\bar{g}_{i j}=g_{i j}
$$

After the above normalization, we have

$$
\bar{h}^{\prime}=\left(d^{2} \bar{A}_{0}^{\prime}, \bar{C}_{n}^{\prime}\right)=\frac{1}{\sigma}\left(d^{2} \bar{A}_{0}, \bar{C}_{n}\right)=\frac{1}{\sigma} \bar{h}=h .
$$

It follows that

$$
\bar{h}_{i j}=h_{i j} .
$$

Note that in (27) and (28) we wrote $\bar{g}_{i j}$ and $\bar{h}_{i j}$ instead of $\bar{g}_{i j}^{\prime}$ and $\bar{h}_{i j}^{\prime}$.

Taking the exterior derivatives of equations (24), we obtain

$$
\left[\bar{\omega}_{j}^{i}-\omega_{j}^{i}-\delta_{j}^{i}\left(\bar{\omega}_{0}^{0}-\omega_{0}^{0}\right)\right] \wedge \omega^{j}=0 .
$$


Applying Cartan's lemma to these equations, we find that

$$
\bar{\omega}_{j}^{i}-\omega_{j}^{i}=\delta_{j}^{i}\left(\bar{\omega}_{0}^{0}-\omega_{0}^{0}\right)+T_{j k}^{i} \omega^{k},
$$

where $T_{j k}^{i}=T_{k j}^{i}$. It is easy to prove that the quantities $T_{j k}^{i}$ form a $(1,2)$-tensor, which is called the deformation tensor of the tangent bundle.

Differentiating equations (27), we obtain

$$
g_{i k}\left(\bar{\omega}_{j}^{k}-\omega_{j}^{k}\right)+g_{k j}\left(\bar{\omega}_{i}^{k}-\omega_{i}^{k}\right)=0 .
$$

Substituting for $\bar{\omega}_{j}^{i}-\omega_{j}^{i}$ the values taken from (29), we find that

$$
2 g_{i j}\left(\bar{\omega}_{0}^{0}-\omega_{0}^{0}\right)+\left(g_{i k} T_{j l}^{k}+g_{k j} T_{i l}^{k}\right) \omega^{l}=0 .
$$

It follows that the 1 -form $\bar{\omega}_{0}^{0}-\omega_{0}^{0}$ is expressed in terms of the basis forms $\omega^{l}$ :

$$
\bar{\omega}_{0}^{0}-\omega_{0}^{0}=s_{l} \omega^{l} .
$$

Next, we make the transformation

$$
\bar{A}_{i}^{\prime}=\bar{A}_{i}+x_{i} \bar{A}_{0}
$$

in the pencil of normal hyperspheres. Since $\bar{A}_{0}^{\prime}=\bar{A}_{0}$, we have

$$
d \bar{A}_{0}^{\prime}=\bar{\omega}_{0}^{0} \bar{A}_{0}+\omega^{i} \bar{A}_{i}=\bar{\omega}_{0}^{0} \bar{A}_{0}+\omega^{i}\left(\bar{A}_{i}^{\prime}-x_{i} \bar{A}_{0}\right) .
$$

It follows that

$$
{ }^{\prime} \bar{\omega}_{0}^{0}=\bar{\omega}_{0}^{0}-x_{i} \omega^{i}
$$

By (31), from this we find that

$$
\bar{\omega}_{0}^{0}=\omega_{0}^{0}+\left(s_{i}-x_{i}\right) \omega^{i} .
$$

We can see now that by setting $x_{i}=s_{i}$, we reduce relation (31) to the form

$$
\bar{\omega}_{0}^{0}=\omega_{0}^{0} .
$$

By (32), equations (30) take the form

$$
g_{i l} T_{j k}^{l}+g_{j l} T_{i k}^{l}=0
$$

By cycling these equations with respect to the indices $i, j$ and $k$ and subtracting the first equation from the sum of the last two equations, we obtain the conditions

$$
T_{i j}^{k}=0,
$$

by means of which equations (29) become

$$
\bar{\omega}_{j}^{i}=\omega_{j}^{i} .
$$

Taking the exterior derivatives of equations (32), we obtain the exterior quadratic equation

$$
\left(\bar{\omega}_{i}^{0}-\omega_{i}^{0}\right) \wedge \omega^{i}=0,
$$

from which, by Cartan's lemma, it follows that

$$
\bar{\omega}_{i}^{0}-\omega_{i}^{0}=t_{i j} \omega^{j}, \quad t_{i j}=t_{j i} .
$$

Taking the exterior derivatives of (33), we obtain

$$
\omega^{i} \wedge\left(\bar{\omega}_{j}^{0}-\omega_{j}^{0}\right)+\bar{\omega}_{n}^{i} \wedge \bar{\omega}_{j}^{n}-\omega_{n}^{i} \wedge \omega_{j}^{n}+g^{i k} g_{j l}\left(\bar{\omega}_{k}^{0}-\omega_{k}^{0}\right) \wedge \omega^{l}=0 .
$$


By (28), the second and third terms on the left-hand side cancel out. Substituting for $\bar{\omega}_{i}^{0}-\omega_{i}^{0}$ in the remaining terms the values taken from (34), and using the fact that the forms $\omega^{l}$ are linearly independent, we find that

$$
-t_{j k} \delta_{l}^{i}+t_{j l} \delta_{k}^{i}+g^{i m}\left(t_{m k} g_{j l}-t_{m l} g_{j k}\right)=0 .
$$

Contracting this relation with respect to the indices $i$ and $k$, we arrive at the equation

$$
(n-3) t_{j l}=-t g_{j l},
$$

where $t=g^{i m} t_{i m}$. Since $n \geq 4$, by contracting the latter equation with the tensor $g^{j l}$, we find that $(2 n-4) t=0$. It follows that $t=0$, and consequently $t_{j l}=0$.

As a result, equation (34) takes the form

$$
\bar{\omega}_{i}^{0}=\omega_{i}^{0} .
$$

From equations (14) it follows that

$$
\bar{\omega}_{i}^{n}=\omega_{i}^{n} .
$$

Taking the exterior derivatives of equations (37), we obtain

$$
\omega_{i}^{n+1} \wedge\left(\bar{\omega}_{n}^{0}-\omega_{n}^{0}\right)=0 .
$$

By (7), even for $n \geq 3$, the forms $\omega_{i}^{n+1}$ are linearly independent, and as a result, we find that

$$
\bar{\omega}_{n}^{0}=\omega_{n}^{0}
$$

Exterior differentiation of equations (36) and (39) leads to the identity. Thus the system of equations (23), (24), (27), (32), (33), (36) (37) and (39) is completely integrable.

Moreover, equations (23), (24), (27), (32), (33), (36) (37) and (39) show that all components of an infinitesimal displacement of second order moving frames associated with the hypersurfaces $V^{n-1}$ and $\bar{V}^{n-1}$ coincide. Thus, by the equivalence theorem of É. Cartan (see [3] or [9], the hypersurface $\bar{V}^{n-1}$ can be obtained from the hypersurface $V^{n-1}$ by means of a conformal transformation. Therefore, the hypersurfaces $V^{n-1}$ and $\bar{V}^{n-1}$ are conformally equivalent.

As we can see from equation (35), the proof of our main theorem fails if $n=3$. To prove the rigidity theorem for $n=3$, it is necessary to add certain additional conditions to condition (20) which are connected with a third order differential neighborhood. Such a theorem for $n=3$ was proved in [12].

\section{REFERENCES}

1. M. A. Akivis and V. V. Goldberg, Projective differential geometry of submanifolds. NorthHolland, Amsterdam-New York-Tokyo, 1993, xi+362 pp. MR 94i:53001

2. M. do Carmo, and M. Dajczer, Conformal rigidity. Amer. J. Math. 109 (1987), no. 5, 963-985. MR 89e:53016

3. É. Cartan, Les sous-groupes des groupes continus de transformations. Ann. Sci. École Norm. (3) 25 (1908), 57-194. Jbuch. 39, pp. 206-207

4. L_ La déformation des hypersurfaces dans l'espace conforme réel à $n \geq 5$ dimensions. Bull. Soc. Math. France 45 (1917), 57-121. Jbuch. 46, p. 1129

5. 259-356. Jbuch. 47, pp. 656-657

6. G. Fubini, Applicabilitá proiettiva di due superficie. Rend. Circ. Mat Palermo 41 (1916), 135-162. Jbuch. 46, pp. 1098-1099 
7. _ Studî relativi all' elemento lineare proiettivo di una ipersuperficie. Atti Accad. Naz. Lincei Rend. Cl. Sci. Fis. Mat. Natur. (5) 27 (1918), 99-106. Jbuch. 46, p. 1095

8. G. Fubini and E. Čech, Geometria proiettiva differenziale. Zanichelli, Bologna, vol. 1, 1926, 394 pp., vol. 2, 1927, 400 pp. Jbuch. 52, pp. 751-752

9. R. Gardner, The method of equivalence and its applications. CBMS-NSF Regional Conference Series in Applied Mathematics, 58. SIAM, Philadelphia, PA, 1989, vii+127 pp. MR 91j:58007

10. G. R. Jensen and E. Musso, Rigidity of hypersurfaces in complex projective space, Ann. Sci. École Norm. Sup. (4) 27 (1994), 227-248. MR 95a:53020

11. R. Sacksteder, The rigidity of hypersurfaces, J. Math. Mekh. 11 (1962), 929-940. MR 26:1833

12. C. Schiemangk, and R. Sulanke, Submanifolds of the Möbius space, Math. Nachr. 96 (1980), 165-183. MR 82d:53017

13. R. Sulanke, R: Submanifolds of the Möbius space. III The analogue of O. Bonnet's theorem for hypersurfaces. Tensor (N. S.) 38 (1982), 311-317. MR 87m:53066

Department of Mathematics, Ben-Gurion University of the Negev, P.O. Box 653, BeER-Sheva 84105, IsRael

E-mail address: akivis@black.bgu.ac.il

Department of Mathematics, New Jersey Institute of Technology, University Heights, Newark, New Jersey 07102

E-mail address: vlgold@numerics.njit.edu 